\title{
The heat-stable antigen determines pathogenicity of self-reactive $T$ cells in experimental autoimmune encephalomyelitis
}

\author{
Xue-Feng Bai, Jin-Qing Liu, Xingluo Liu, Yong Guo, Karen Cox, Jing Wen, \\ Pan Zheng, and Yang Liu \\ Department of Pathology and Comprehensive Cancer Center, The Ohio State University Medical Center, \\ Columbus, Ohio, USA \\ Yong Guo's present address is Aventis Pharmaceuticals, CNS-Molecular Biology, Bridgewater, New Jersey, USA. \\ Received for publication November 30, 1999, and accepted in revised form March 14, 2000.
}

Address correspondence to: Yang Liu,Department of Pathology, Ohio State University Medical Center, 129 Hamilton Hall, 1645 Neil Avenue, Columbus, Ohio 43210, USA. Phone: (614) 292 3054; Fax (614) 688-8152; E-mail: liu-3@medctr.osu.edu.

Induction of myelin-specific CD4 T cells is a pivotal event in the development of experimental autoimmune encephalomyelitis (EAE). Other checkpoints in EAE pathogenesis have not been clearly defined, although multiple genetic loci are known to influence EAE development. We report here that targeted mutation of the heat-stable antigen (HSA) abrogates development of EAE despite a complete lack of effect on induction of autoimmune T cells. To test whether T-cell expression of HSA is sufficient, we created transgenic mice in which HSA is expressed exclusively in the T-cell lineage. We found that these mice remain resistant to EAE induction. Adoptive transfer studies demonstrate that both $\mathrm{T}$ cells and non-T cells must express HSA in order for the pathogenic $\mathrm{T}$ cells to execute their effector function. Moreover, HSAIg, a fusion protein consisting of the extracellular domain of the HSA and the Fc portion of immunoglobulin, drastically ameliorates the clinical sign of EAE even when administrated after self-reactive $T$ cells had been expanded. Thus, identification of HSA as a novel checkpoint, even after activation and expansion of self-reactive $T$ cells, provides a novel approach for immunotherapy of autoimmune neurologic diseases, such as multiple sclerosis.

J. Clin. Invest. 105:1227-1232 (2000).

\section{Introduction}

Experimental autoimmune encephalomyelitis (EAE) is mediated primarily by CD4 T cells specific for autoantigens in the central nervous system (CNS) (1-3). After induction, the pathogenic T cells must migrate to the CNS to initiate the inflammatory process, which is responsible for the clinical signs of EAE. Although activation requirements and properties of self-reactive CD4 T cells have been extensively investigated (4-8), the mechanisms for subsequent steps, such as trafficking of autoreactive $\mathrm{T}$ cells to the CNS and the effector function of T cells in the CNS, have not been clearly understood. For instance, P- and Eselectins, which are essential for target organ-localization of T cells to other tissues (9), are not required for the development of $\operatorname{EAE}(10)$.

The heat-stable antigen (HSA) was initially identified as a marker for differentiation of hematopoietic (11, 12) and neuronal cells (13). Accumulating data indicate that the HSA provides a CD28-independent costimulation for clonal expansion and functional differentiation of both CD4 and CD8 T cells (14-20). The wide expression of HSA, however, argues that it may play other roles in immunity. Interestingly, although HSA disappears from the $\mathrm{T}$ cell surface as $\mathrm{T}$ cells mature (21), activation of $\mathrm{T}$ cells leads to a rapid induction of
HSA $(22,23)$. The function of HSA on T cells has not been clearly elucidated, although anti-HSA mAb can enhance proliferation of $\mathrm{T}$ cells in response to stimulation with anti-CD3 and anti-CD28 (23). Here, we report that targeted mutation of the HSA abrogates induction of EAE without affecting the induction of autoreactive $T$ cells and that the development of EAE requires expression of HSA on both $T$ cells and non- $T$ host cells. Our results reveal a novel checkpoint for the development of EAE.

\section{Methods}

Mice. Wild-type (WT) C57BL/6 mice were purchased from the National Cancer Institute (Bethesda, Maryland, USA). Mice homozygous for the disrupted HSA (produced with embryonic stem cells from C57BL/6 mice) $(18,24)$ or CD28 (25) (backcrossed to C57BL/6 for more than eight generations) locus have been described before and are maintained at the animal facilities of the Ohio State University Medical Center. HSA transgenic mice (HSATG) have been described previously (22) and have been backcrossed to $\mathrm{C} 57 \mathrm{BL} / 6 \mathrm{j}$ background for more than five generations. Mice with HSA exclusively expressed on the T-cell lineage (HSATG/HSA ${ }^{-/}$) were generated by crossing HSATG with the $\mathrm{HSA}^{-/-}$mice. 
Induction and clinical evaluation of EAE. The immunogen, myelin oligodendrocyte glycoprotein (MOG) peptide 3555 of rat origin (MEVGWYRSPFSRVVHLYRNGK), was synthesized by Research Genetics, Inc. (Huntsville, Alabama, USA). The purity of the peptide was greater than $90 \%$. Mice of 8-12 weeks of age were immunized subcutaneously with $200 \mu \mathrm{g}$ MOG peptide in CFA (400 $\mu \mathrm{g}$ of Mycobacterium tuberculosis per milliliter) in a total volume of $100 \mu \mathrm{L}$. They received $200 \mathrm{ng}$ of pertussis toxin (List Biological, Campbell, California, USA) in 200 $\mu \mathrm{L}$ PBS in the tail vein immediately after the immunization and again 48 hours later. The mice were observed every other day and scored on a scale of $0-5$ with gradations of 0.5 for intermediate scores: 0 , no clinical signs; 1 , loss of tail tone; 2 , wobbly gait; 3 , hind limb paralysis; 4 , hind and fore limb paralysis; and 5 , death.

T-cell proliferation assay. Draining lymph node cells were isolated 10 days after immunization. A total of $5 \times 10^{5}$ cells per well were stimulated with given concentrations of MOG peptide in the presence $6 \times 10^{5}$ cells per well of irradiated (20 Gy) syngeneic splenocytes for 60 hours. The cultures were pulsed with $\left[{ }^{3} \mathrm{H}\right]$ thymidine $(1 \mu \mathrm{Ci} /$ well; ICN Pharmaceuticals Inc., Costa Mesa, California, USA) for another 12 hours, and incorporation of $\left[{ }^{3} \mathrm{H}\right]$ thymidine was measured in a liquid scintillation $\beta$-plate counter.

ELISpot assay to evaluate frequencies of T cells that produce IFN- $\gamma$, IL-2, and IL-4 upon restimulation with MOG peptide in vitro. The antibody pairs and the procedures have been described (20), except that the MOG peptide was used for stimulation at $10 \mu \mathrm{g} / \mathrm{mL}$. The numbers presented are those of cytokine producers per million of draining lymph node cells.

Histology. Mice were sacrificed by $\mathrm{CO}_{2}$ inhalation. Spinal cords were removed by insufflation and fixed in $10 \%$ formalin/PBS. Paraffin sections were prepared and stained with hematoxylin and eosin. Neurologic lesions were graded on each of the 10 cross sections per spinal cord, according the following criteria: 0 , no infiltrate; 1 , three or less focal meningeal infiltrates; 2 , more than three focal meningeal infiltrates; 3 , up to five perivascular infiltrate foci in the parenchyma with involvement of less than $5 \%$ of the white matter; 4 , five to ten perivascular foci in the parenchyma or invasions involving $5-25 \%$ the white matter; 5 , more than ten perivascular foci or diffuse infiltration involving more than $25 \%$ of the white matter.

Passive transfer of EAE. Groups of eight to ten WT and $\mathrm{HSA}^{-/}$mice were immunized with $200 \mu \mathrm{g}$ of MOG peptide subcutaneously. At 10 days after immunization, draining lymph nodes were harvested and stimulated at $4 \times 10^{6} / \mathrm{mL}$ in Click's EHAA medium supplemented with $15 \% \mathrm{FCS}, 5 \% \mathrm{IL}-2$ supernatant, and $50 \mu \mathrm{g} / \mathrm{mL}$ of MOG peptide for 4 days. A total of $1 \times 10^{8}$ cells were injected intraperitoneally into each recipient mouse that had been $\gamma$-irradiated (5.5 Gy) 1 hour earlier.

Preparation of fusion protein and treatment of EAE. The HSA fragment encoding the signal peptide and the mature protein sequence were amplified by PCR, using GGA AAG CTT ATG GGC AGA GC as forward primer, CGA GAT CTC TGG TGG TAG CG as reverse primer, and HSA cDNA as template. The PCR products were digested with Hind III and $B g l$ II enzymes and were ligated to Hind III- and Xba I-digested pCDM8 vector (Invitrogen Corp., San Diego, USA) and a Xba I- and Bam HI-treated DNA fragment encoding human IgG1 Fc, which were amplified by PCR using CAG GGA TCC CGA GGG TGA GTA CTA AGC TAG CTT CAG CGC TCC TGC CTG as forward primer, CTT CGA CCA GTC TAG AAG CAT CCT CGT GCG ACC GCG AGA GC as reverse primer, and
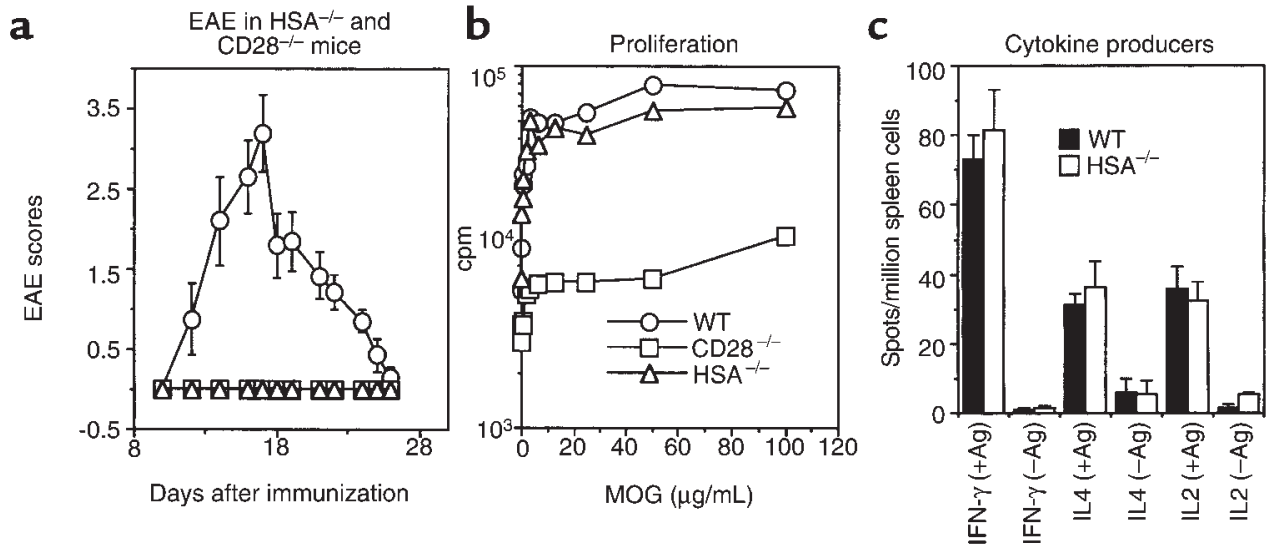

\section{Figure 1}

Targeted mutations of HSA and CD28 reveal two distinct checkpoints in the development of EAE. (a) Targeted mutations of either HSA or CD28 prevent induction of EAE. WT, CD28 $8^{--}$, or $\mathrm{HSA}^{-/}$mice were immunized with MOG peptide. Clinical signs were scored as described in Methods. (b) Proliferative response of lymph node T cells to MOG peptides. Draining lymph node cells from day 10-immunized mice were stimulated with given concentrations of MOG peptide and irradiated syngeneic naive spleen cells as antigen-presenting cells. (c) Enumeration of cytokine-producers by ELISpot. Draining lymph node cells used in $\mathbf{b}$ were used as responder cells. The numbers of cells secreting either IL2, IL4, or IFN- $\gamma$ among $1 \times 10^{6}$ lymph node cells in response to MOG peptide (AA35-55) are presented. Data shown are means \pm SEM from three independent experiments. 

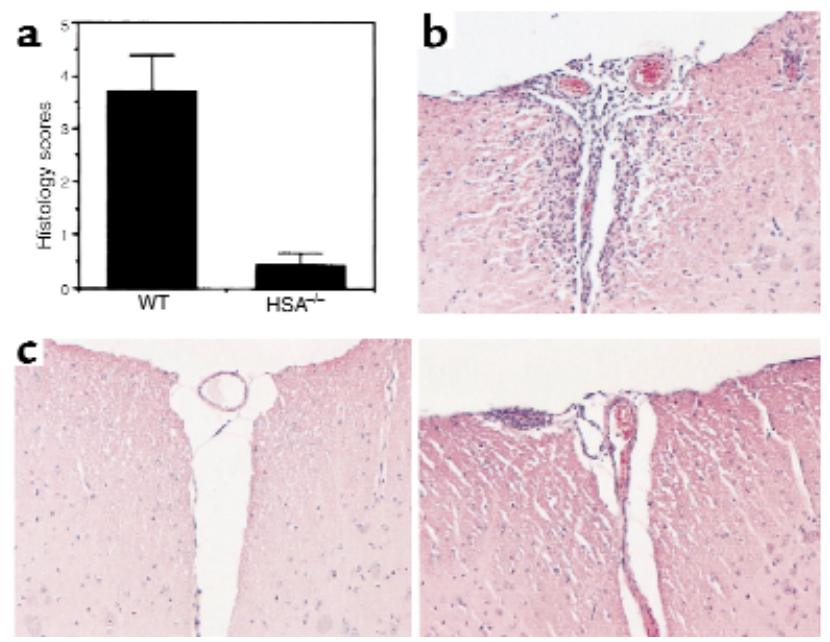

\section{Figure 2}

Histological analysis of spinal cord of MOG-immunized WT or $\mathrm{HSA}^{-/}$mice. (a) The means and SEM of histological scores of WT and $\mathrm{HSA}^{-/-}$mice spinal cords. Ten independent cross sections, from cervical to sacral regions, were examined in each spinal cord. The data are summarized from 30 spinal cord sections from three mice in each group. (b) Representative histology in immunized WT mice; all sections examined contain histology lesions. (c) Histology sections $(\times 100)$ of immunized $\mathrm{HSA}^{-/-}$mice. A lesion-free section is presented on the left, and a lesion-containing section is presented on the right.

DNA from human peripheral blood as template. The construct was verified by DNA sequencing and was used to transfect the Chinese Hamster Ovary cell line. The cells that secreted HSAIg fusion protein were amplified in DMEM containing 5\% FCS until confluence. The cell monolayers were washed with serumfree medium and cultured in optimal $\mathrm{M}$ medium for 72 hours. The supernatants were collected and the HSAIg was purified using a protein-G column according to the manufacturer's protocol. The purity of the protein was verified by SDS-PAGE.

\section{Results}

To test whether HSA is essential for the development of EAE, we immunized C57BL/6 WT, and HSA- or CD28deficient mice with MOG peptide AA35-55 in conjunction with CFA and pertussis toxin. As shown in Figure 1a, WT mice developed acute EAE within 2 weeks of peptide immunization, whereas those with targeted mutation of either HSA or CD28 were completely resistant to EAE induction. The requirement for $\mathrm{CD} 28$ in EAE induction is consistent with previous reports $(26,27)$. Interestingly, although targeted mutation of CD28 ablated induction of MOG-specific T cells, as revealed by proliferative response of draining lymph node cells, that of HSA had little effect on peptide-specific T-cell proliferation (Figure 1b). Moreover, the frequencies of antigen-specific, IL2-, IL4-, and IFN- $\gamma$-producing cells were not altered in $\mathrm{HSA}^{-/-}$mice (Figure 1c). The anti-MOG peptide IgG responses were also detected in HSA-deficient mice (data not shown). The normal T-cell responses in $\mathrm{HSA}^{-/-}$mice are consistent with our previous observations that targeted mutation of HSA alone is insufficient to prevent induction of T-cell responses $(18,20)$. The differential effects of HSA and CD28 mutations on T-cell priming reveal that these genes mediate two distinct checkpoints in the development of EAE: CD28 controls induction of autoreactive T cells, whereas HSA determines their pathogenicity.

Histological analysis of MOG peptide immunized WT and HSA confirms the clinical scores. The histological scores are summarized in Figure 2a, whereas representative histology sections are presented Figure 2 , b and c. As shown in Figure 2b, active immunization with MOG peptide induces multiple neurologic lesions in the WT mice, characterized by multiple lesions with extensive invasion of parenchyma. In contrast, the spinal cords of $\mathrm{HSA}^{-/}$mice are either devoid of any lesion (Figure 2c, left), or with one or two low-grade lesions involving meninges (Figure 2c, right).
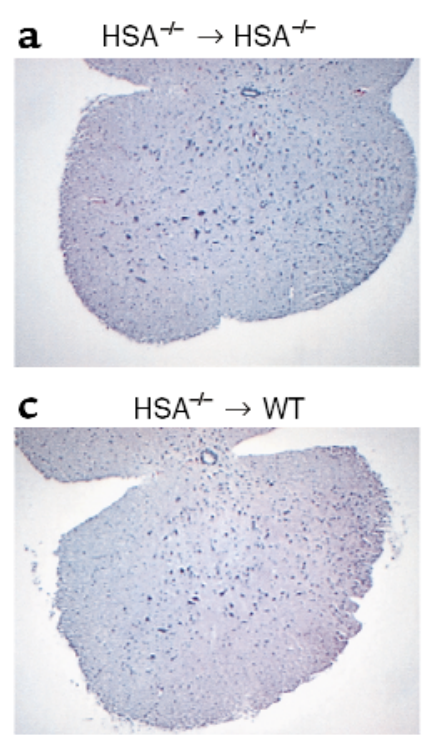
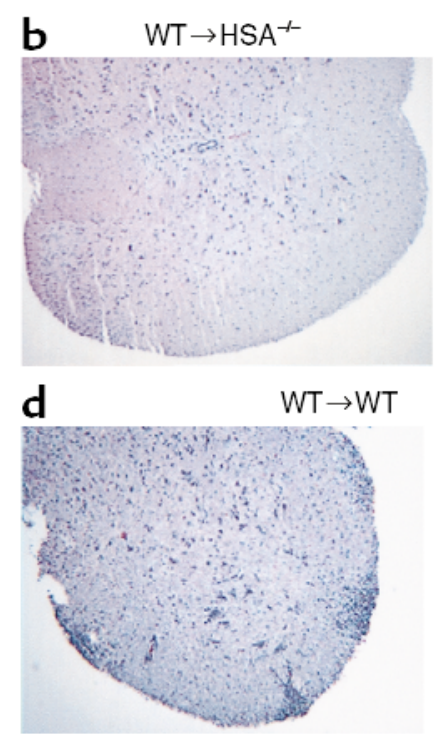

Figure 3

Requirement for HSA expression on both $T$ cells and non-T host cells for the induction of EAE. Histology ( $\times 63$ for $\mathbf{a}-\mathbf{c}$ and the left side of $\mathbf{d} ; \times 200$ for the right side of $\mathbf{d}$ ) of spinal cords of the $\mathrm{HSA}^{-/-}$(a and b) or WT (c and $\mathbf{d}$ ) recipient mice on day 12 after adoptive transfer. Draining lymph node cells were isolated from either WT or $\mathrm{HSA}^{-/}$mice after immunization and were stimulated with antigen and IL-2 for 4 days in vitro. The activated $T$ cells were injected into either WT or $\mathrm{HSA}^{-/-}$mice $\left(10^{8}\right.$ cells per mouse). EAE development was monitored daily for clinical signs. At 12 days after transfer, recipient mice were sacrificed and spinal cords were processed for histological examination. No disease was observed in $\mathrm{WT} \rightarrow \mathrm{HSA}^{-/-}, \mathrm{HSA}^{-/-} \rightarrow \mathrm{WT}$, or $\mathrm{HSA}^{-/}$ $\rightarrow \mathrm{HSA}^{-/-}$recipients. 


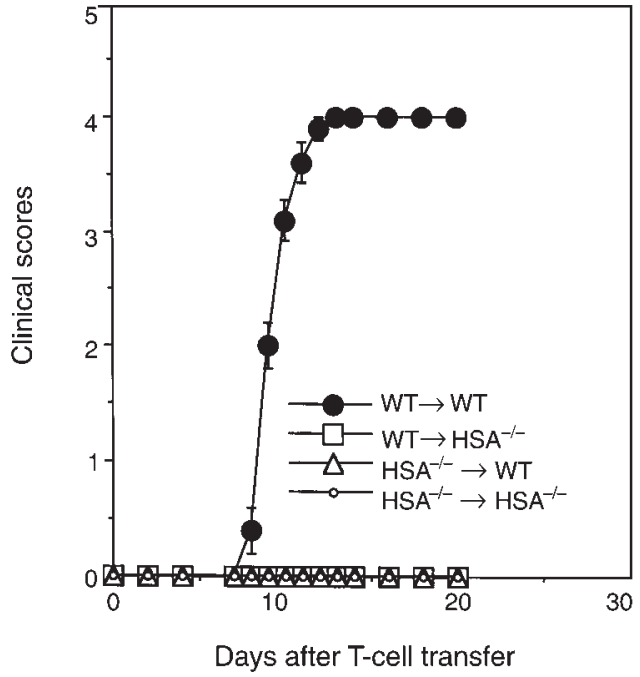

Figure 4

Clinical scores of the adoptive transfer experiment with four (WT $\rightarrow$ $\mathrm{HSA}^{-/-}$and $\mathrm{HSA}^{-/-} \rightarrow$ WT groups) or five (WT $\rightarrow \mathrm{WT}^{-}$and $\mathrm{HSA}^{-/-} \rightarrow$ $\mathrm{HSA}^{-/}$groups) mice per group.

Because HSA is expressed on activated T cells and non-T host cells of hematopoietic and neuronal lineages $(12,13,22)$, it is of interest to determine whether HSA expressed on $T$ cells is necessary and/or sufficient for EAE development. We adoptively transferred activated draining lymph node cells to WT and HSA-deficient recipients. As shown in Figure 3 and Figure 4, WT $\mathrm{T}$ cells induced severe EAE in WT recipients within 8 days of adoptive transfer. Interestingly, none of the HSA-deficient recipients developed EAE. Thus HSA expression on $\mathrm{T}$ cells alone appears insufficient for $\mathrm{EAE}$ development. Moreover, T cells from HSA-deficient mice failed to induce disease regardless of HSA gene status in the recipient, which indicates that HSA expression on $\mathrm{T}$ cells is necessary for EAE development. These results strongly suggested that HSA must be expressed on both host cells and autoreactive $T$ cells in order to induce EAE.

To substantiate these observations, we produced mice that expressed HSA exclusively on $\mathrm{T}$ cells. We have previously reported the transgenic mice in which expression of HSA was under the control of the lck proximal promoter (HSATG) (22). For this study, we crossed the HSA transgene to HSA-deficient mice to produce mice that expressed HSA exclusively on T cells (Figure 5a). To test whether HSA expression on the T-cell lineage is sufficient for EAE development, we immunized WT, HSATG, $\mathrm{HSA}^{-/-}$, and HSATG HSA ${ }^{-/-}$mice with MOG peptide. As shown in Figure 5b, WT and HSATG mice developed EAE with essentially identical kinetics, which indicates that transgenic expression of HSA on T cells does not prevent the production and effector function of self-reactive T cells. Nevertheless, much like $\mathrm{HSA}^{-/}$ mice, the mice with exclusive HSA expression on the Tcell lineage failed to develop EAE. These results demonstrated clearly that HSA expression on T-cell lineage alone is insufficient for EAE development.

That HSA may be a critical checkpoint after activation of self-reactive $T$ cells suggests a novel approach in treating autoimmune neurologic diseases. Given that antiHSA mAb was toxic in the EAE model, to address this issue, we produced a fusion protein between the extracellular domain of HSA and the Fc portion of human IgG1, to block the HSA-mediated interactions (data not shown). As shown in Figure 6a, the fusion protein has an apparent molecular weight of about $100 \mathrm{kDa}$ under nonreducing SDS-PAGE. After reduction, it migrated as a $50-\mathrm{kDa}$ band. Thus, HSAIg is likely a homodimer, as expected. We treated mice starting at 8-10 days after
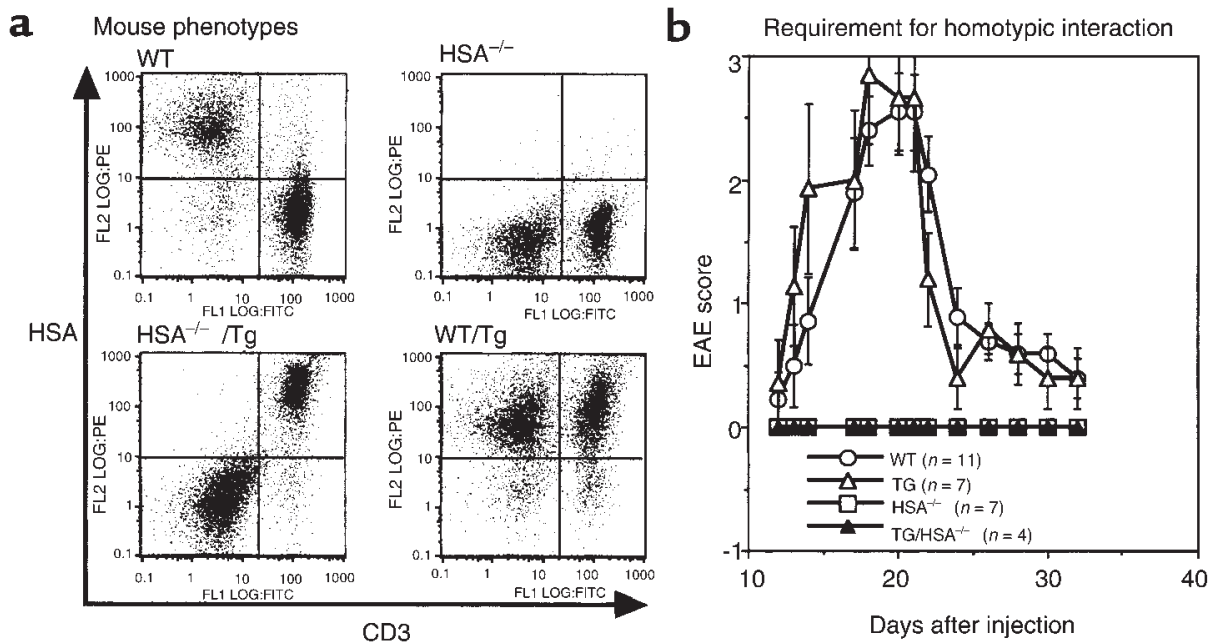

Figure 5

Transgenic expression of HSA exclusively on T-cell lineage is insufficient for EAE development. (a) Phenotypes of WT, HSA-TG, HSA $/$, , and

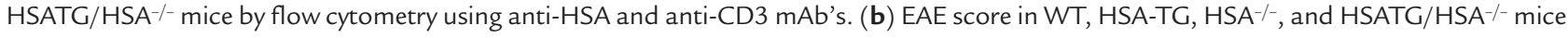
after immunization with the MOG peptides. 
immunization with MOG peptide, when MOG-specific T-cell response had already expanded in the local lymph nodes. As shown in Figure 6b, HSAIg drastically ameliorated EAE. All HSAIg-treated mice recovered substantially earlier than did the control mice. Because MOG-reactive T cells had been activated before HSAIg administration, the clinical signs in the treated group may reflect the fact that some autoreactive T cells had already migrated into the CNS.

\section{Discussion}

We report here that targeted mutation of HSA abrogated the development of EAE. These results demonstrate that HSA is a critical EAE checkpoint. Consistent with our previous observations in priming of CD4 and CD8 $\mathrm{T}$ cells specific for virus and foreign antigens $(18,20)$, targeted mutation in HSA alone has no effect in induction MOG-specific CD4 T cells, as measured by either cytokine profiles or by in vitro recall proliferative response. Thus, the HSA checkpoint resides after T-cell activation.

Results from adoptive transfer and transgenic mice with exclusive HSA expression in T-cell lineage demonstrate that HSA must be expressed on both T cells and non-T host cells. Many lineages of hematopoietic and neuronal cells express HSA (13), and the type of non-T host cells that need to express HSA for EAE development remains to be identified.

The data presented here reveal a novel function of HSA in EAE after induction of self-reactive T cells. Following are several mechanisms that can be invoked to explain the role of HSA.

First, recent studies from this and other laboratories revealed that costimulatory molecules, such as B7, play a critical role at both induction and effector phases of antitumor immunity (28-31) and autoimmunity (32-34). Because HSA is a costimulatory molecule, it is of interest to consider whether HSA acted as a costimulatory molecule at the effector phase in the development of EAE, much as B7 does (34). We consider this unlikely because costimulation by HSA does not require HSA expression on T cells (22), whereas HSA expression on T cells is essential for the development of EAE.

Second, the substantial reduction in inflammation in HSA-deficient mice after MOG immunization favors the notion that HSA may be involved in T-cell trafficking to the CNS. Because HSA is known to interact with P-selectin (35), one hypothesis is that HSA controls EAE development by interacting with Pselectin. This is very unlikely, as P-and E-selectins are not required for EAE development (10). Moreover, VLA4 binding to VCAM-1 or fibronectin appears essential for T-cell trafficking to the CNS in EAE (4). However, HSA is not required for induction of VLA4 in autoreactive $\mathrm{T}$ cells in vivo after immunization (data not shown). Nevertheless, it has been reported that HSA can enhance VLA4 interaction with VCAM1 and fibronectin (36). Since VLA4 is essential for T-
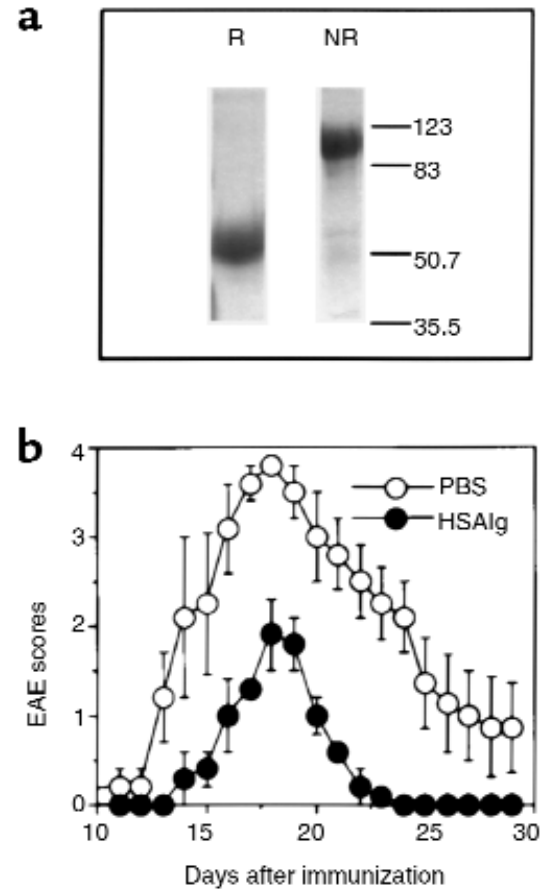

Figure 6

HSAlg ameliorates EAE. (a) Analysis of HSAlg by SDS-PAGE. A total of $10 \mu \mathrm{g}$ of purified HSAlg was separated by $10 \%$ reducing $(R)$ and nonreducing (NR) SDS-PAGE. The proteins were stained by Coomassie blue. (b) The EAE score for control (PBS) or HSAlg-treated mice. EAE was induced in WT mice as described in Methods. On days $8,10,12,14$, and 22 after immunization, five mice per group were injected intraperitoneally with $100 \mu \mathrm{g} /$ mouse of either HSAlg or $100 \mu \mathrm{L}$ of PBS as control. The effect of HSAlg has been evaluated in three independent experiments with similar results.

cell trafficking to CNS, HSA may facilitate this process. Finally, HSA can interact with itself in a homotypic interaction (37). Because the HSA is expressed at high levels on the vascular endothelial cells in the CNS (data not shown), we suggest that homotypic interaction between HSA on T cells and HSA on vascular endothelial cells may be critical for migration of self-reactive T cells to the CNS.

Regardless of the precise function of HSA in EAE, identification of HSA as a novel checkpoint in EAE developments may provide a new window for immune intervention of autoimmune neurologic disease. This concept is supported by our observation that soluble HSA drastically ameliorated EAE after autoreactive T cells had been primed. Given the presence of autoreactive $T$ cells in autoimmune patients, HSA can be a valuable therapeutic target for neurologic autoimmune diseases, such as multiple sclerosis.

\section{Acknowledgments}

We thank P.J. Nielsen for HSA-deficient mice; C. Whitacre and M. Caligiuri for critical reading of the manuscript; and J. Kiel for secretarial assistance. This study is supported by a grant from the National Institutes of Health (AI32981). 
1. Mendel, I., Kerlero de Rosbo, N., and Ben-Nun, A. 1995. A myelin oligodendrocyte glycoprotein peptide induces typical chronic experimental autoimmune encephalomyelitis in $\mathrm{H}-2 \mathrm{~b}$ mice: fine specificity and $\mathrm{T}$ cell receptor $\mathrm{V}$ beta expression of encephalitogenic T cells. Eur. J. Immunol. 25:1951-1959.

2. Kerlero de Rosbo, N., Mendel, I., and Ben-Nun, A. 1995. Chronic relapsing experimental autoimmune encephalomyelitis with a delayed onset and an atypical clinical course, induced in PL/J mice by myelin oligodendrocyte glycoprotein (MOG)-derived peptide: preliminary analysis of MOG T cell epitopes. Eur. J. Immunol. 25:985-993.

3. Zamvil, S.S., and Steinman, L. 1990. The T lymphocyte in experimental allergic encephalomyelitis. Annu Rev. Immunol. 8:579-621.

4. Baron, J.L., Madri, J.A., Ruddle, N.H., Hashim, G., and Janeway, C.A., Jr. 1993. Surface expression of alpha 4 integrin by CD4 T cells is required for their entry into brain parenchyma. J. Exp. Med. 177:57-68.

5. Vanderlugt, C.L., et al. 1998. The functional significance of epitope spreading and its regulation by co-stimulatory molecules. Immunol. Rev. 164:63-72.

6. Miller, R.A., Garcia, G., Kirk, C.J., and Witkowski, J.M. 1997. Early activation defects in $\mathrm{T}$ lymphocytes from aged mice. Immunol. Rev. 160:79-90.

7. Miller, S.D., et al. 1995. Evolution of the T-cell repertoire during the course of experimental immune-mediated demyelinating diseases. Immunol. Rev. 144:225-244.

8. Lafaille, J.J., et al. 1997. Myelin basic protein-specific T helper 2 (Th2) cells cause experimental autoimmune encephalomyelitis in immunodeficient hosts rather than protect them from the disease. J. Exp. Med. 186:307-312.

9. Springer, T.A. 1994. Traffic signals for lymphocyte recirculation and leukocyte emigration: the multistep paradigm. Cell. 76: 301-314.

10. Engelhardt, B., Vestweber, D., Hallmann, R., and Schulz, M. 1997. E- and $\mathrm{P}$-selectin are not involved in the recruitment of inflammatory cells across the blood-brain barrier in experimental autoimmune encephalomyelitis. Blood. 90:4459-4472.

11. Springer, T., Galfre, G., Secher, D.S., and Milstein, C. 1978. Monoclonal xenogeneic antibodies to murine cell surface antigens: identification of novel leukocyte differentiation antigens. Eur. J. Immunol. 8:539-551.

12. Bruce, J., Symington, F.W., McKearn, T.J., and Sprent, J. 1981. A monoclonal antibody discriminating between subsets of $\mathrm{T}$ and $\mathrm{B}$ cells. $J$. Immunol. 127:2496-2501.

13. Rougon, G., Alterman, L.A., Dennis, K., Guo, X.J., and Kinnon, C. 1991. The murine heat-stable antigen: a differentiation antigen expressed in both the hematolymphoid and neural cell lineages. Eur. J. Immunol. 21:1397-1402.

14. Liu, Y., et al. 1992. Co-stimulation of murine CD4 T cell growth: cooperation between B7 and heat-stable antigen. Eur. J. Immunol. 22:2855-2859.

15. Liu, Y., et al. 1992. Heat-stable antigen is a costimulatory molecule for CD4 T cell growth. J. Exp. Med. 175: 437-445.

16. Enk, A.H., and Katz, S.I. 1994. Heat-stable antigen is an important costimulatory molecule on epidermal Langerhans' cells. J. Immunol. 152:3264-3270

17. De Bruijn, M.L., Peterson, P.A., and Jackson, M.R. 1996. Induction of heat-stable antigen expression by phagocytosis is involved in in vitro activation of unprimed CTL by macrophages. J. Immunol. 156:2686-2692.

18. Liu, Y., Wenger, R.H., Zhao, M., and Nielsen, P.J. 1997. Distinct costimulatory molecules are required for the induction of effector and memory cytotoxic T lymphocytes. J. Exp. Med. 185:251-262.

19. Wang, Y.-C., Zhu, L., McHugh, R., Sell, K.W., and Selvaraj, P. 1995.
Expression of heat-stable antigen on tumor cells provides co-stimulation for tumor-specific T cell proliferation and cytotoxicity in mice. Eur. J. Immunol. 25:1163-1167.

20. Wu, Y., Zhou, Q., Zheng, P., and Liu, Y. 1998. CD28-independent induction of $T$ helper cells and immunoglobulin class switches requires costimulation by the heat-stable antigen. J. Exp. Med. 187:1151-1156.

21. Crispe, I.N., and Bevan, M.J. 1987. Expression and functional significance of the J11d marker on mouse thymocytes. J. Immunol. 138:2013-2018.

22.Zhou, Q., Wu, Y., Nielsen, P.J., and Liu, Y. 1997. Homotypic interaction of the heat-stable antigen is not responsible for its co-stimulatory activity for T cell clonal expansion. Eur. J. Immunol. 27:2524-2528.

23. Hubbe, M., and Altevogt, P. 1994. Heat-stable antigen/CD24 on mouse T lymphocytes: evidence for a costimulatory function. Eur. J. Immunol. 24:731-737.

24. Nielsen, P.J., et al. 1997. Altered erythrocytes and a leaky block in B-cell development in CD24/HSA-deficient mice. Blood. 89:1058-1067.

25. Shahinian, A., et al. 1993. Differential T cell costimulatory requirements in CD28-deficient mice. Science. 261:609-612.

26. Oliveira-dos-Santos, A.J., et al. 1999. CD28 costimulation is crucial for the development of spontaneous autoimmune encephalomyelitis. $J$. Immunol. 162:4490-4495.

27. Shi, F.D., et al. 1998. Differential requirements for CD28 and CD40 ligand in the induction of experimental autoimmune myasthenia gravis. Eur. J. Immunol. 28:3587-3593.

28. Chong, H., Hutchinson, G., Hart, I.R., and Vile, R.G. 1998. Expression of B7 co-stimulatory molecules by B16 melanoma results in a natural killer cell-dependent local anti-tumour response, but induces T-cell-dependent systemic immunity only against B7-expressing tumours. Br. J. Cancer. 78:1043-1050

29. Ramarathinam, L., Castle, M., Wu, Y., and Liu, Y. 1994. T cell costimulation by B7/BB1 induces CD8 $\mathrm{T}$ cell-dependent tumor rejection: an important role of B7/BB1 in the induction, recruitment, and effector function of antitumor T cells. J. Exp. Med. 179:1205-1214.

30. Wu, T.C., Huang, A.Y., Jaffee, E.M., Levitsky, H.I., and Pardoll, D.M. 1995. A reassessment of the role of B7-1 expression in tumor rejection. J. Exp. Med. 182:1415-1421.

31. Sarma, S., et al. 1999. Cytotoxic T lymphocytes to an unmutated tumor rejection antigen P1A: normal development but restrained effector function in vivo. J. Exp. Med. 189:811-820.

32. Allison, J., et al. 1998. The threshold for autoimmune T cell killing is influenced by B7-1. Eur. J. Immunol. 28:949-960.

33. Soldevila, G., Geiger, T., and Flavell, R.A. 1995. Breaking immunologic ignorance to an antigenic peptide of simian virus 40 large $\mathrm{T}$ antigen. $J$. Immunol. 155:5590-5600.

34. Chang, T.T., Jabs, C., Sobel, R.A., Kuchroo, V.K., and Sharpe, A.H. 1999. Studies in B7-deficient mice reveal a critical role for B7 costimulation in both induction and effector phases of experimental autoimmune encephalomyelitis. J. Exp. Med. 190:733-740.

35. Aigner, S., et al. 1995. Heat stable antigen (mouse CD24) supports myeloid cell binding to endothelial and platelet P-selectin. Int. Immunol. 7:1557-1565.

36. Hahne, M., Wenger, R.H., Vestweber, D., and Nielsen, P.J. 1994. The heatstable antigen can alter very late antigen 4-mediated adhesion. J. Exp. Med. 179:1391-1395.

37. Kadmon, G., Eckert, M., Sammar, M., Schachner, M., and Altevogt, P. 1992. Nectadrin, the heat-stable antigen, is a cell adhesion molecule. J. Cell Biol. 118:1245-1258 